\title{
CICLO PDCA COMO FERRAMENTA DA QUALIDADE PARA A MELHORIA EM SERVIÇOS
}

\section{PDCA CYCLE A QUALITY TOOL FOR IMPROVEMENT IN SERVICES}

\author{
Gabrieli Marina Ricci - gabi.marina14@gmail.com \\ Faculdade de Tecnologia de Taquaritinga - Taquaritinga - São Paulo - Brasil \\ Rosemary Chiuchi Magrini - mary_magrini@yahoo.com.br \\ Faculdade de Tecnologia de Taquaritinga - Taquaritinga - São Paulo - Brasil \\ Marcos Alberto Claudio Pandolfi-marcos.pandolfi@fatectq.edu.br \\ Faculdade de Tecnologia de Taquaritinga - Taquaritinga - São Paulo - Brasil
}

DOI: 10.31510/infa.v18i1.1122

Data de submissão: 16/04/2021

Data do aceite: 09/07/2021

Data da publicação: 30/07/2021

\begin{abstract}
RESUMO
O objetivo deste artigo foi buscar conhecimento do ciclo PDCA (Plan, Do, Check, Action) aplicado na qualidade de serviços. Para tanto fez-se uma revisão de bibliográfica. Adotou-se a metodologia descritiva onde observou, registrou e analisou. Os dados bibliográficos foram levantados em SCIELO ( Scientific Electronic Library Online), Revistas online: Sociedade \& Natureza, USP, FGV, PPGEA/FURG, Ambiente \& Sociedade Biblioteca Brasileira de Teses e Dissertações. A pesquisa foi realizada de maio a agosto de 2020, com publicações dos últimos 10 anos e foi estruturada com referencial teórico. Onde foi possível finalizar dizendo que, com o entendimento sobre o PDCA, ficou claro, que a qualidade é conjunto de técnicas e procedimentos administrativos, composta por ferramentas que tem o objetivo auxiliar todos os setores de uma organização e de acordo com literatura o PDCA é uma ferramenta que traz contribuições em vários aspectos para incrementar as discussões sobre da qualidade e, a partir da percepção de que sua estrutura inclui requisitos essenciais como: conceito de qualidade; combinação das abordagens estatística e estratégica.
\end{abstract}

Palavras-chave: PDCA. Qualidade. Serviços.

\begin{abstract}
The objective of this article was to seek knowledge of the PDCA cycle (Plan, Do, Check, Action) applied to the quality of services. To this end, a bibliographic review was carried out. The descriptive methodology was adopted, where it observed, registered, and analyzed. Bibliographic data were collected in SCIELO (Scientific Electronic Library Online), Online magazines: Society \& Nature, Environment \& Society Brazilian Library of Theses and
\end{abstract}


Dissertations. The research was carried out from May to August 2020, with publications from the last 10 years and was structured with a theoretical framework. Where it was possible to conclude by saying that, with the understanding of the PDCA, it was clear that quality is a set of administrative techniques and procedures, composed of tools that have the objective of helping all sectors of an organization and, according to literature, the PDCA is a tool that brings contributions in several aspects to increase discussions about quality and, based on the perception that its structure includes essential requirements such as: concept of quality; combination of statistical and strategic approaches.

Keywords: PDCA. Quality. Services.

\section{INTRODUÇÃO}

De acordo com Instituto Brasileiro de Geografia e Estatística (IBGE) tendo como base as pesquisas voltadas às estatísticas sociais, demográficas e econômicas, o setor de serviços no ano de 2019 teve uma alta de 3,3\%, com o crescimento expressivo no setor de prestação de serviços aliada à alta competitividade dos mercados, às empresas buscam constantemente a qualidade de seus serviços e sua diferenciação na satisfação dos clientes. De modo geral a qualidade na prestação de serviço é muito questionada pelos consumidores.

Segundo Dias (2011) os consumidores não compram somente aquilo de que necessitam, mas compram serviços e produtos que aparentemente, de acordo com a mídia, possui o efeito de acrescentar qualidades que deseja ter.

Kotler e Keller (2012) salientam que o setor de serviços é a complexidade interna da empresa e a incerteza externa do mercado, que podem se tornar impedimentos para que a organização se oriente pelo mercado. As empresas que tendem ser bem-sucedida devem ter a capacidade de realizar atividades com qualidade pensando sempre no cliente.

Nesse contexto, justifica esta pesquisa para compreender de que maneira o ciclo PDCA pode auxiliar no controle de qualidade de serviços. Para tanto a pergunta norteadora para o estudo é como o ciclo PDCA melhora a qualidade do serviço?

Considera-se relevante o estudo do tema PDCA devido à sua importante contextualização no setor de serviços. O tema deste estudo está delimitado entre a teoria e o discurso do ciclo PDCA, ferramenta aplicada na qualidade. O tema foi escolhido por estar em evidência no meio empresarial, acadêmico e ao mesmo tempo, se firmado como uma eficiente prática na busca da melhoria contínua em produtos/serviços.

Objetivo desse artigo é estudar a aplicação do ciclo o PDCA no que se refere ao gerenciamento da qualidade no setor de serviços. A metodologia adotada par o estudo é uma revisão de literatura de cunho bibliográfico. 


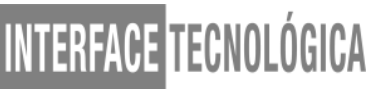

Este trabalho foi divido em seções, sendo está a introdução, a segunda seção o referencial teórico, a qualidade e conceitos correlatos, definições de serviços e o ciclo PDCA. $\mathrm{Na}$ terceira seção apresenta-se a metodologia de pesquisa. Na quarta seção os resultados e discussões. Na última seção (quinta) seguem as considerações finais do estudo.

\section{QUALIDADE E CONCEITOS CORRELATOS}

A qualidade consiste na conformidade com os requisitos e devem estar aliadas em conjunto e características que atenda as exigências do cliente, quando equilibrada, essa relação se traduz em clientes satisfeitos e sucesso nos negócios (PMI, 2013).

Segundo Werkema (2012) as políticas de qualidade envolvem estratégicas com prioridades em conjunto de normas, métodos e procedimentos visando a satisfação do cliente.

Paladini (2012) conceitua a qualidade como sendo um conjunto de ações operacionais, centradas e localizadas em pequenas melhorias do produto/serviços e deve ser vista como um dos elementos fundamentais do gerenciamento das organizações. De modo geral o conceito da qualidade vai muito além do controle de qualidade dos produtos e serviços de uma empresa, mas também traz o conceito de gerenciamento para alcance da eficiência e eficácia nas operações.

\subsection{Definições de Serviços}

Paladini (2012, p. 332) define serviços como sendo "atividades de consumo, é campo de interação entre fornecedor e cliente ocorre de forma de fluxo entre tempo e espaço".

Zeithaml, Bitner e Gremler (2011, p. 39) conceituam serviços como "atos, processos e atuações oferecidos ou coproduzidos por uma entidade ou pessoa, para outra entidade ou pessoa”. Segundo Dias (2011, p. 106) serviço é um “produto intangível, podendo ser entendido como uma ação ou um desempenho que cria valor por meio de uma mudança desejada no cliente ou em seu benefício".

Para Las Casas (2010) para fornecer serviços com qualidade é necessário que os fornecedores tenham uma sequência de procedimentos como pesquisar, desenvolver uma cultura, ter treinamento e estabelecer mecanismos de controle. Destarte, se o resultado de uma prestação de serviços iguala a expectativa, o cliente fica satisfeito. 


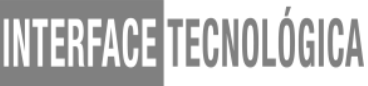

\subsection{Ciclo PDCA - Plan, Do, Check, Action}

De acordo com Moen e Norman (2007) o PDCA (Plan, Do, Check, Action) do portugues (Planejar, Fazer, Checar, Agir) deu encadeamento intermitente para a fabricação de um produto e expandiu até se tornar um conceito genérico e aplicável a todos os processos dentro de uma organização.

O ciclo PDCA ficou conhecido no Japão nos anos 50. após ser levado por Deming, Shewhart que percebeu que seu modelo também era aplicável para processos repetitivos de melhoria de atividades de planejamento. O ciclo de Deming foi criado por Walter A. Shewart, em 1920, mas ficou mundialmente conhecido como ciclo de Deming (MOEN; NORMAN, 2007).

Para Carpinetti, (2012. p.14) o "PDCA pode ser aplicado em todas as áreas funcionais de uma organização, nas quais podem ser identificadas as funções da administrativa”.

Em termos teóricos, o ciclo PDCA tem promovido mudanças e benefícios significativos, aprimoramento da qualidade, pois incorpora vários métodos, cada um voltado para uma finalidade específica, soluciona problemas e melhoria de resultados (CARPINETTI, 2012).

O PDCA é um método amplamente aplicado para o controle eficaz e confiável das atividades de uma organização, principalmente aquelas relacionadas às melhorias, possibilitando a padronização nas informações de controle de qualidade e a menor probabilidade de erros nas análises ao tornar as informações mais claras (GONÇALEZ, 2015).

De modo geral o ciclo PDCA pode ser interpretado como um método dinâmico que auxilia a resolver problemas, e consequentemente, tomar decisões de modo organizado, potencializado por meio de ações.

\section{PROCEDIMENTOS METODOLÓGICOS}

Uma pesquisa cientifica tem o objetivo de adquirir conhecimento por meio de uma investigação sistemática onde se procura respostas para soluções de problemas. A metodologia científica é chegar à verdade por meio da dúvida sistemática e da decomposição do problema em pequenas partes, características que definiram a base da pesquisa científica.

De acordo com Rampazo (2005, p. 53) “a pesquisa descritiva observa, registra, analisa e correlaciona fatos ou fenômenos (variáveis), sem manipulá-los; estuda fatos e fenômenos do mundo físico e, especialmente, do mundo humano, sem a interferência do pesquisador. 


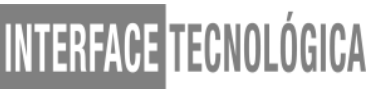

Gil (2006, p. 145) diz que a "escolha de um tema de pesquisa, bem com a sua realização, necessariamente é um ato político".

A metodologia adotada nesta pesquisa é uma abordagem descritiva. Para o levantamento dos dados foram utilizadas SCIELO (A Scientific Electronic Library Online), Revistas online: Sociedade \& Natureza, USP, FGV, PPGEA/FURG, Ambiente \& Sociedade Biblioteca Brasileira de Teses e Dissertações. A pesquisa foi realizada no período de agosto de 2020 a março de 2021, com publicações dos últimos 10 anos e está estruturada com referencial teórico. Baseou-se a pesquisa nas palavras-chave: Qualidade; Serviços; PDCA. As bases de dados foram elencadas 30 publicações que abordavam a terma. Sendo que foram selecionadas 10 para a análise e interpretação do conteúdo, considerando o objetivo do tema proposto.

\section{RESULTADOS E DISCUSSÃO}

Propôs-se nesta pesquisa bibliográfica a qual se objetivou buscar a compreensão de que forma o ciclo o PDCA é utilizado no gerenciamento da qualidade no setor de serviços.

Procurando por meio dos dados da pesquisa em vários autores destacar a opinião individual de cada um sobre o assunto. Foram consultados periódicos livros e dissertações que descrevem a aplicação do ciclo PDCA. Portanto a pesquisa conseguiu evidenciar quanto, aos conceitos da qualidade, os termos de serviços e o ciclo PDCA.

Nesta etapa, constituída pelo processo de codificação, interpretação e análise do conteúdo encontrado em cada texto, buscando aspectos convergentes e divergentes no discurso dos autores, elencando as informações relevantes que atenderam ao propósito da pesquisa. Das 10 (dez) publicação selecionadas 7 foram exclusas após a leitura na integra ,3(três) publicações de casos práticos foram selecionados para uma análise diagnostica comprovando eficácia da aplicação do Ciclo PDCA. Em seguida os dados foram categorizados e os estudos apresentados em forma de quadro considerando as características dos artigos (Título dos artigos, Autores Base /ano, revista, local de publicação). 


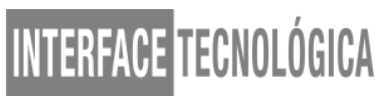

Quadro 1- Fontes consultadas, conteúdos relevantes e autores

\begin{tabular}{|c|c|c|c|}
\hline & Título & Autores & Base/Ano \\
\hline 1 & $\begin{array}{l}\text { A utilização do ciclo } \\
\text { PDCA para melhoria da } \\
\text { qualidade de serviços na } \\
\text { manutenção de shuts. }\end{array}$ & $\begin{array}{l}\text { - Alyson da Luz Pereira } \\
\text { Rodrigues } \\
\text { - Mariene Silva Santos } \\
\text { - Mayanne Camara Serra } \\
\text { - Eduardo Mendonça } \\
\text { Pinheiro }\end{array}$ & $\begin{array}{l}\text { Iberoamerican Journal of Industrial } \\
\text { Engineering, Florianópolis, SC, } \\
\text { Brasil, v.9, n. 18, p. 48-70, } 2017\end{array}$ \\
\hline 2 & $\begin{array}{l}\text { O ciclo PDCA para a } \\
\text { melhoria da qualidade } \\
\text { dos serviços de consulta } \\
\text { em uma unidade de saúde } \\
\text { de Belém do Pará. }\end{array}$ & $\begin{array}{l}\text { Amanda Tavares Cardoso } \\
\text {-Isadora Luzia O. de Quadros } \\
\text {-Jaqueline Marisa de Moura - } \\
\text { Mônica Rocha Paulo } \\
\text {-Nilcely Pricila O. da Silva }\end{array}$ & $\begin{array}{l}\text { VII Simpósio de Excelências em } \\
\text { Gestão e Tecnologia- SEGET - } \\
2016\end{array}$ \\
\hline 3 & $\begin{array}{l}\text { Aplicação de ferramentas } \\
\text { da qualidade no método } \\
\text { PDCA para melhoria } \\
\text { contínua: estudo de caso } \\
\text { numa empresa fabricante } \\
\text { de autopeças. }\end{array}$ & $\begin{array}{l}\text {-Maicon Bruno Ralene } \\
\text { de Melo } \\
\text {-Maria Bernadete Pinto } \\
\text {-Diego Jean de Melo } \\
\text {-Jorge Nei Brito }\end{array}$ & $\begin{array}{l}\text { Os Desafios da Engenharia na Era } \\
\text { da Inovação Anais do XV } \\
\text { CONEMI - Congresso Nacional de } \\
\text { Engenharia Mecânica e Industrial } \\
\text { IX SEEMI - Seminário Estadual de } \\
\text { Engenharia Mecânica e Industrial } \\
.2017 \text {. }\end{array}$ \\
\hline
\end{tabular}

Fonte: Próprio autor

Os artigos selecionados retratam a aplicação do PDCA de forma ampla destacando outras ferramentas que auxiliam a aplicação do PDCA para a melhoria da qualidade de serviços.

No primeiro artigo analisado os autores Rodrigues et al. (2017) aplicaram o ciclo PDCA visando a qualidade nos serviços de manutenção em uma indústria de mineração. Para a aplicação do ciclo PDCA, tiveram como apoio métodos de Diagrama de Ishikawa e análise dos 5 Porquês.

Segundo Slack et al. (2018) o Ishikawa Diagrama é um método efetivo de ajudar a se encontrar as causas raízes do problema. O diagrama inicia-se tendo em vista o efeito que pode ser um problema e os 5 porquês é um método que permite por meio de múltiplos questionamentos onde separe a causa do efeito, contribuindo para a construção de hipóteses plausíveis para a causa raiz do problema. 


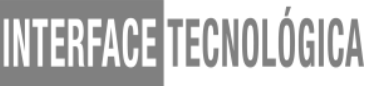

Segundo Rodrigues et al. (2017) com a utilizando o Diagrama de Ishikawa, foi possível identificar o problema, e com os 5 Porquês ficou evidenciado a possibilidade de melhoria controle da aplicação de inspeções preditivas no setor de manutenções no ambiente estudado.

$\mathrm{Na}$ análise do segundo artigo Cardoso et al. (2016) utilizaram como ferramentas de apreciação, a Análise de Pareto, o Diagrama de Ishikawa, e 5W2H como forma de identificar as principais causas dos problemas quanto à qualidade no atendimento na unidade de saúde.

Para Polacinski (2012) a ferramenta 5W2H abrange todo um plano de ação para atividades pré-estabelecidas, tem por finalidade mapeamento das atividades e a definição das ações a serem tomadas, qual forma serão realizadas e quem irá executar as atividades. De modo geral o cerne da ferramenta 5W2H é responder as questões de forma organizada.

Cardoso et al (2016) constataram com o PDCA que o atendimento ao público no Posto de Saúde é abaixo do esperado, gerando insatisfação, pelos usuários. Os autores observaram que a infraestrutura é insuficiente para desempenhar os serviços de qualidade.

No terceiro artigo analisado Melo et al. (2017) iniciaram o estudo sugerido por Carpinetti (2010) de identificar os problemas prioritários, coletar os dados, fazer uma análise, buscar as causas-raízes, planejar e implementar as ações para finalmente apurar os resultados.

De acordo Melo et al. (2017) observaram que a aplicação das ferramentas Diagrama de Ishikawa e a técnica 5W2H assim como as ciclo do PDCA são simples elementos que não dependem de um conhecimento técnico específico para serem aplicados, permitindo a utilização por toda a organização, promovendo a disseminação da cultura de melhoria contínua a todos os níveis e concluíram que o ciclo PDCA é prático e eficaz, apontando etapas a serem seguidas para que seja possível identificar os prováveis problemas, priorizá-los, buscar soluções e padronizá-los.

\section{CONSIDERAÇÕES FINAIS}

O objetivo artigo foi de buscar conhecimento do ciclo PDCA aplicado na qualidade de serviços. Para atingir o objetivo proposto consistiu em uma seleção de publicações, buscando complementaridades, confirmações, que tratavam de definições dos objetos de estudo, dos pressupostos, das formulações teóricas dos estudos para as questões de interesse.

Ressalta-se que a importância dos usos das ferramentas da qualidade no ciclo PDCA, pois cada uma delas possui sua significância. Sem elas não seria possível a realização do PDCA. 


\section{WWTEREFGEETECNOLÓGICA}

Nos três casos após a aplicação do PDCA foi possível a identificação dos problemas, sendo possível saná-los.

Pode-se finalizar esta pesquisa dizendo que, com o entendimento sobre o PDCA, ficou claro que a qualidade é conjunto de técnicas e procedimentos administrativos, composta por ferramentas que tem o objetivo auxiliar todos os setores de uma organização. De acordo com literatura o PDCA é uma ferramenta que traz contribuições em vários aspectos para incrementar as discussões sobre da qualidade e, a partir da percepção de que sua estrutura inclui requisitos essenciais como: conceito de qualidade; combinação das abordagens estatística e estratégica. Considera-se, portanto, que foram alcançados os objetivos traçados para este estudo.

\section{REFERÊNCIAS}

CARDOSO; Amanda Tavares; QUADROS, Isadora Luzia O de; MOURA, Jaqueline Marisa de; PAULO, Mônica Rocha; SILVA, Nilcely Pricila O. da. O ciclo PDCA para a melhoria da qualidade dos serviços de consulta em uma unidade de saúde de Belém do Pará. VII Simpósio de Excelências em Gestão e Tecnologia- SEGET -2016. Disponível em: https://www.aedb.br/seget/arquivos/artigos10/216. Acesso em maio de 2020.

CARPINETTI, Luiz Cesar Ribeiro. Gestão da Qualidade: Conceitos e Técnicas. 2. Ed. São Paulo: Atlas, 2012.

DIAS, Sergio Roberto. Gestão de marketing. 2 ${ }^{\mathrm{a}}$ Ed. 2011 São Paulo: Saraiva 2011.

GIL, Antônio Carlos. Métodos e técnicas de pesquisa social. 5. ed. São Paulo: Atlas, 2006.

GONÇALEZ, Patrícia Ueda; Comparação dos índices de capacidade do processo para distribuições não normais. Revista Gestão e Produção, v 16, n. 01, p 121-132, 2015.

IBGE- Instituto Brasileiro de Geografia e Estatística. Pesquisa Anual dos Serviços. 2019. Disponível em: https://www.ibge.gov.br/home/estatistica/economia/comercioeservico. Acesso em jul.de 2020.

KOTLER, Philip; KELLER, Kevin Lane. Administração de Marketing - 14ª ed. Pearson 2012.

LAS CASAS, Alexandre Luzzi. Marketing de Serviços. 5ª ed. São Paulo, 2010.

LOVELOCK, Christopher; WIRTZ, Jochen; HEMZO, Miguel Angelo. Marketing de Serviços: Pessoas, Tecnologias e Estratégia. São Paulo: Pearson, 2011.

MELO, Maicon Bruno Ralene de; PINTO Maria Bernadete; MELO Diego Jean de; BRITO Jorge Nei. Aplicação de ferramentas da qualidade no método PDCA para melhoria contínua: estudo de caso numa empresa fabricante de autopeças. Os Desafios da 
Engenharia na Era da Inovação Anais do XV CONEMI - Congresso Nacional de Engenharia Mecânica e Industrial IX SEEMI - Seminário Estadual de Engenharia Mecânica e Industrial .2017 .

MOEN, Ron; NORMAN, Cliff. Evolution of the PDSA Cycle. Disponível em http://deming.ces.clemson.edu/PDF. Acesso em 20 de dez. de 2020.

PALADINI Edson Pacheco. Gestão da qualidade: Teoria e Prática - $3^{\mathrm{a}}$ ed. Atlas 2012.

PMI - Project Management Institute- Gerenciamento de projetos Guia PMBOK Campus Boulevard 2013.

RAMPAZZO, Lino. Metodologia científica: para alunos dos cursos de graduação e pósgraduação. 3. ed. São Paulo:Loyola, 2005.

RODRIGUES, Alyson da Luz Pereira; SANTOS, Mariene Silva; SERRA, Mayanne Camara; PINHEIRO, Eduardo Mendonça. A utilização do ciclo PDCA para melhoria da qualidade de serviços na manutenção de shuts. Iberoamerican Journal of Industrial Engineering, Florianópolis, SC, Brasil, 0v.9, n. 18, p. 48-70, 2017. Disponível em: http://incubadora.periodicos.ufsc.br/index.php/IJIE/article/view/v9n1803/pdf. Acsso em jun de 2020 .

SLACK, Nigel.; BRANDON-JONES, Alistair; JOHNSTON, Robert. Administração da Produção. $8^{\text {a }}$ ed. Atlas São Paulo, 2018.

WERKEMA, Maria Cristina Catarino Criando a cultura Lean Six Sigma. Rio de Janeiro: Elsevier, 2012.

ZEITHAML, Valarie ABITNER, Mary Jo.; GREMLER, Dwayne D. Marketing de serviços: a empresa com foco no cliente. 5. ed. Porto Alegre: Bookman, 2011. 